\title{
Interpretationsphilosophie
}

\section{Kommentare und Repliken}

\author{
Von GÜNTER ABEL (Berlin)
}

In den vorliegenden Beiträgen des Symposiums zu dem Buch „Interpretationswelten“ wird eine Vielzahl interessanter und wichtiger Aspekte erörtert. Die folgenden Bemerkungen und Erwiderungen können sich nur auf einige dieser Punkte beziehen. Diese Konzentration erfolgt in der Hoffnung, die Anliegen der an der Diskussion Beteiligten nicht zu verfehlen, Mißverständnisse zu beseitigen und zugleich grundlegende Aspekte der Interpretationsphilosophie zu verdeutlichen.

\section{Zu Josef Simon}

Der Beitrag von Josef Simon ${ }^{1}$ hat Anliegen, Reichweite, systematischen Zusammenhang und Grundcharakter der „Interpretationswelten“ mit überaus anregenden Diskussionspunkten kommentiert.

\section{Einwand des Relativismus}

Sprachlichen und/oder nicht-linguistischen Zeichen ist ihre Bedeutung weder vorab und definitiv eingebaut, noch ist sie beliebig, d.h. gänzlich von den subjektiven Meinungen der einzelnen Sprecher und Hörer abhängig. Ersteres wäre Essentialismus oder Magie der Zeichen; letzteres dagegen relativistische Beliebigkeit (mit seiner extremsten Form im Solipsismus). Weder das eine noch das andere ist jedoch für gelingende Verständigungsverhältnisse kennzeichnend. Die Bedeutung der Zeichen wird, so die interpretationsphilosophische These, durch deren angemessene Interpretation bestimmt. Wenn demgemäß jedes sich in Zeichen vollziehende Sprechen, Denken und Handeln stets nur innerhalb eines Interpretations-Horizonts und einer Interpretations-Praxis möglich ist, dann heißt dies auch, daß, wie Simon (855) mit Recht betont, das interpretierende Ich in diese Prozesse ,eingebunden", mithin an der Beliebigkeit seiner Vorstellungen gehindert ist. Dies läßt sich im Rekurs auf das Stufenmodell der Interpretation erläutern. Jeder individuelle Sprach- und Zeichengebrauch, der auf der dritten Ebene der Interpretation erfolgt, ist schon deshalb nicht beliebig, weil er bereits unter Bedingungen der zweiten und der ersten Ebene der Interpretation steht (im Beispiel: unter der Bedingung einer gegebenen Sprache, z.B. dem

1 Welten und Ebenen, im vorliegenden Heft der Deutschen Zeitschrift für Philosophie, $855 \mathrm{ff}$. 
Deutschen, und unserer sich in Zeichen vollziehenden Weise, sin der Welt< zu sein). Mit Hilfe des Stufenmodells der Interpretation kann man auch den Unterschied und das Verhältnis zwischen Sprecher-Bedeutung und semantischer Bedeutung verständlich machen. Umgekehrt bedeutet dies jedoch keine Determination und keine Garantie für gelingende Verständigung auf der dritten Ebene. Vor dem Hintergrund der zentralen Stellung der Interpretations-Praxis ${ }^{2}$ handelt es sich um eine interpretations-praxeologische Relativität ohne Relativismus, nicht um einen Relativismus der Beliebigkeit unter Restriktionen.

Der Interpretationismus betont nachdrücklich, daß die relativistische These, jede Interpretation sei so gut wie jede andere, offenkundig nicht in der Lage ist, den sprach-, zeichen- und handlungsphilosophisch entscheidenden Punkt, die gelingende Verständigung, verständlich zu machen. Zwar läßt sich die Idee absoluter Synonymie zwischen zwei und mehreren Zeichen nicht einlösen. Aber dies bedeutet eben nicht, daß Beliebigkeit der Bedeutung eines Zeichens sowie seiner Gleichsetzung mit anderen Zeichen gegeben ist. Damit käme „kein Mensch zurecht“ (863). Mithin steht, wie Simon formuliert, gegen den Relativismus „die Vernünftigkeit im Sprachgebrauch, d.h. der Wille zur Kommunikabilität“. Zugleich ist darin auch der Essentialismus der Verständigung ausgeschlossen, demzufolge wir uns ,, in unseren Explikationen nach einem $>$ Wesen der Dinge $<$ richten müßten, um unserer Sprache Bestimmtheit zu geben“ (ebd.). Ein solches Ansinnen würde gelingende Verständigungsverhältnisse, würde Kommunikabilität eher zerstören als befördern.

\section{Logos der Interpretationszeichen}

Mit dem Aspekt, daß alles, was man sagen kann, stets nur aus einem Interpretations-Horizont und aus einer Interpretations-Praxis (Lebenspraxis; Lebensform) heraus und auf diese hin gesagt werden kann, kommen zwei (eng zusammengehörige) Punkte ins Spiel, die, wie schon für Kant und Wittgenstein, so auch für den interpretationsphilosophischen Ansatz von grundlegender Bedeutung sind.

Erstens (a) gilt im Sinne Wittgensteins, daß ein Bild der Wirk lichkeit seine eigene „Form der Abbildung ... nicht abbilden (kann); es weist sie auf."3

Zweitens (b) ist hinsichtlich der in die Tiefe gestaffelten Interpretations-Praxis der Unterschied zu betonen zwischen der Deutung eines Zeichens und dem ssich erfüllenden Voll$z u g$ reines Zeichens als Zeichen. Handelt es sich um den Zeichenvollzug, dann hat dieser die Bedingungen seines Gelingens nicht in etwas anderem außerhalb seiner selbst. Es geht mithin um den Unterschied zwischen dem > Vollzug resp. Verstehen des Zeichens $\prec$ und der >Deutung resp. Erklärung des Zeichens $\varsigma$. Die Deutung ist von dem Vollzug in dem Sinne verschieden, daß an dem Punkt, an dem ein Zeichen verstanden wird, Deutungen gerade nicht entscheidend sind. Wenn ich ein Zeichen verstehe, dann deute und erkläre ich es nicht; ich verstehe es. Das aber heißt: Wir sind im Verwenden und Verstehen mit dem Vollzugscharakter der Zeichen, mit dem Logos der sprachlichen und/oder nicht-linguistischen Zeichen einerseits gut, ja bestens vertraut und bekannt (insofern wir ihn, mit Hegel gesprochen, fortwährend ,im Munde“ führen); andererseits jedoch - und das ist hier die

2 Vgl. dazu Verf., Interpretation und Realität. Erläuterungen zur Interpretationsphilosophie, in: Allgemeine Zeitschrift für Philosophie, 21 (1996), 286 ff.

3 L. Wittgenstein, Tractatus, Nr. 2.172; bei S, 8, Abs. 1. 
Pointe der Rede vom Logos bzw. vom Logischen der Interpretationszeichen - bedeutet dies gerade nicht, daß wir damit das Logische des Zeichens nach Art eines Gegenstandes auch serkannt ‘ hätten. Denn das Logische, der Logos des sprachlichen Zeichens, ist nicht etwas Erkennbares, ist kein innerweltlicher Gegenstand der Erkenntnis und des Aussprechens, sondern Bedingung von deren Möglichkeit. Die logische Form szeigt siche „darin, daß wir >in< ihr denken, ohne sie selbst denken zu können“ (857). Der Logos der Zeichen ist, so könnte man sagen, in diesem Sinne ineffabile und elusiven Charakters. Und er ist auch nicht relativistisch bezweifelbar. Denn er tritt gar nicht innerweltlich auf, sondern >zeigt sich< als Grenze der Welt und des Sinns. Auf ihn trifft nicht zu, was auf alles, was >in< der Welt als >so-und-so< bestimmt erscheint, zutrifft, nämlich auch anders sein zu können. Daher kann es, aus logischen Gründen, keinen Relativismus des Logischen bzw. des Logos geben. Unter Heranziehung des Stufenmodells der Interpretationsverhältnisse kann man sagen, daß der Deutungscharakter der Zeichen auf der dritten Ebene angesiedelt ist, während der Vollzugs- bzw. Logoscharakter der Zeichen sich an der Form des fraglosen Eingespieltseins unserer Interpretation ${ }_{1}$-Praxis zeigt, die in jedem gelingenden (oder nicht-gelingenden) Zeichengebrauch mitregierend (nicht: determinierend), da' ist und mit der wir, wie betont, bestens bekannt sind, ohne sie damit jedoch auch erkannt zu haben.

\section{Zusammenhang von Logik, Ästhetik und Ethik}

Die Rede von der „Form der Interpretations-Praxis“ als der „Grenze“ der Welt und des Sinns ist nicht auf die logische Form begrenzt. Sie schließt vielmehr die ästhetische und die ethische Form mit ein. Das ist ein kardinaler und konsequenzenreicher Punkt. Simon (855 ff.) betont mit Recht, daß damit bereits auch auf der allgemeinen Ebene der Interpretation $_{1}$-Verhältnisse ästhetische und ethische Begrenzungen (hinsichtlich auch des elusiven Charakters der Semantik erfolgreich verwendeter und verstandener Zeichen) im Spiele sind. Wichtig finde ich den von Simon (857) betonten Aspekt, daß die Interpretationsphilosophie in Sachen Ethik ${ }^{4}$ in der Nähe eher zu Kant als zu Wittgenstein gesehen werden kann. Während Wittgenstein zufolge die Ethik sich „nicht aussprechen läßt“5, sieht Simon, daß es in der Interpretationsphilosophie um „symbolisierende“ Zeichen geht, an denen die Kantische Überlegung festgemacht werden kann, daß uns das Ethische zwar nur „symbolisch“, aber in diesem Sinne eben doch anschaulich zugänglich ist. Das Sinnliche, worin uns das Intelligible, mithin das Ethische, vermittelt wird, ist Kant zufolge das „Schöne“. Dieser Gedankengang läuft auf die Formulierung zu, daß das Schöne „Symbol des Sittlichguten"6 ist. Simon (857) betont, daß Logik, Ästhetik und Ethik auf diese Weise letztlich `dieselben`Grenzen haben, und daß sie erst zusammen eine Welt, meine Welt bilden.

\footnotetext{
$4 \mathrm{Zu}$ den ethischen Konsequenzen des interpretationsphilosophischen Ansatzes vgl. Verf., Interpretationsethik und Demokratie, in: Orientierung in Zeichen. Zeichen und Interpretation III, hg. von J. Simon, Frankfurt/M. 1996, 41-79.
}

5 L. Wittgenstein, Tractatus, Nr. 6.421; S, 3, Abs. 1.

6 I. Kant, Kritik der Urteilskraft, $\$ 59$. 


\section{Verschränkung von Verständigungs- und Weltverhältnissen}

Nachdrücklich zu unterstreichen ist auch (860), daß das Verhältnis zu anderen Personen sowie der Weltbezug unabdingbar Bestandteile gelingender Verständigung darstellen. Wir verstehen einander, wenn wir verstehen, wie die anderen Personen die Zeichen verwenden und sie auf Gegenstände, auf äußere Objekte und Ereignisse beziehen. Für die philosophische Betrachtung dieser Zusammenhänge ist wichtig, daß sich die Kommunikationsund Weltverhältnisse nicht wechselseitig ausstechen, sondern drehtürartig zusammengehören. ${ }^{7}$ Die Fähigkeit, eine semantische Charakterisierung der Ausdrücke und Sätze unserer Sprache zu geben, ist mit der Fähigkeit verbunden, Angaben über die als normal zugrundegelegte Welt (z.B. über Tische und Stühle) machen zu können. Und sofern wir es überhaupt mit einer bestimmten Welt zu tun haben, sind die anderen Personen aufgrund der mit ihnen gemeinsam geteilten Sprache und der öffentlichen Wahrnehmungssituation bereits mit im Spiele.

\section{Pluralität der Welten}

Die Rede von „Interpretationswelten“ verwendet den Ausdruck „Welten“ im Plural. Dies impliziert Simon zufolge (862) noch die Vorstellung „eines $>$ Subjekts $<$ mit uneingeschränkter Übersicht" sowie die einer ,, Übersicht< über verschiedene Welten“ (863). Gegenüber dieser Lesart ist jedoch zu betonen, daß die Pluralität, um die es in der Interpretationsphilosophie geht, nicht als >externe <, sondern als >interne $<$ und darüber hinaus als >konditionale< Pluralität konzipiert ist. ${ }^{8}$ Pluralität kommt als mögliche Alterität auf der Innenseite der Zeichenverwendung und intern mit dem interpretatorischen Charakter eines jeden menschlichen Welt-, Fremd- und Selbstverständnisses auf die Bahn. Jede so-und-so individuierte und damit bestimmte Welt wäre im Falle anderer Individuationsprinzipien eine anders individuierte und in diesem Sinne eine andere Welt. Die Alterität ist intern mit der Bestimmtheit einer Welt gesetzt. Pluralität erscheint daher nicht als synkategorematisch zur einen Welt, sondern als konditional. Ohne sie hätten wir es gar nicht mit einer bestimmten, nicht mit unserer und ein jeder es nicht mit seiner Welt zu tun. Das interpretationistische Konzept der Pluralität rechnet also gerade nicht mit einer Übersicht-vonaußen. Ausgangspunkt ist vielmehr, daß wir von genau dieser Möglichkeit systematisch abgeschnitten sind.

\section{Wahrheitsfrage}

Wenn das, was als ein bestimmter Gegenstand angesehen wird, von dem verwendeten (kategorialisierenden) Sprach- und Interpretationssystem abhängig ist, dann hat dies, wie Simon (864 f.) mit Recht hervorhebt, Konsequenzen auch für den Wahrheitsbegriff. Dieser wird zunächst (a) von der Vorstellung eines externen oder eines Gottesgesichtspunktes befreit. Sodann (b) verliert er seine überlieferte Spitzenstellung, und zwar auch dann, wenn

7 Vgl. Verf., Interpretation und Realität, a.a.O., insbes. 282-286.

8 Vgl. dazu Verf., Interne Pluralität. Sprach- und zeichenphilosophische Grundlagen des theoretischen Pluralismus, in: Dialektik, 1996/3: Pluralismus. Erkenntnistheorie, Ethik und Politik. 
er als ein triviales Konzept aufgefaßt wird, von dem wir guten Gebrauch machen. Simon scheint anzunehmen, daß ich der Wahrheitsproblematik die alte Spitzenstellung erhalten möchte. Dies trifft nicht zu. Zwar wird die Wahrheitsfrage nicht obsolet, und es kommt darauf an, sie mit Hilfe des Stufenmodells der Interpretation einer Reformulierung zuzuführen. ${ }^{9}$ Aber sie verliert ihre Vormachtstellung. In der Tradition hatte der Wahrheitsbegriff nicht zuletzt deshalb eine privilegierte Stellung, weil er nicht nur keiner Explikation bedürftig schien, sondern seinerseits über die Richtigkeit eines Zeichens entschied. Liegt jedoch die Wahrheitsfrage, die ihren Sitz im Urteil hat, innerhalb eines Interpretations-Horizonts und einer Interpretations-Praxis, dann setzt Wahrheit das fraglose Eingespieltsein einer Interpretations-Praxis der Zeichen bereits voraus und nimmt diese in Anspruch.

\section{Zu Logi Gunnarsson}

In den ersten beiden Teilen seines Beitrags gibt Logi Gunnarsson ${ }^{10}$ eine Rekonstruktion wichtiger Aspekte der Interpretationsphilosophie. Im dritten Teil argumentiert er dann für einen Monismus >Der Einen Welt $<$, der gleichwohl die im Interpretationismus formulierten Einsichten, vor allem den Pluralismus der Interpretationen, positiv beibehalten möchte. Während ich den ersten beiden Teilen ohne weiteres zustimmen kann, habe ich in bezug auf den ,interpretationspluralistischen Monismus (IPM)“ Bedenken.

\section{Rekonstruktionen}

Vor allem die folgenden Punkte sieht Gunnarsson richtig und nimmt sie positiv auf: (a) die adualistische Konzeption des Verhältnisses von Geist und Welt; (b) den Sinn der Rede vom interpretativen Charakter des menschlichen Weltverständnisses und die drei Ebenen des Interpretationsmodells (- wobei ich noch einen Aspekt hinzufüge, der die Ausweitung der Rede von „Interpretation“ verständlicher machen kann: wichtig ist, ,interpretativ" in seiner adjektivischen und in seiner adverbialen Verwendungsweise zu nehmen und „Interpretation" als Prädikat aufzufassen; tut man dies, dann macht die Erweiterung von ,,interpretieren/interpretativ" über das "Interpretieren-von-etwas-Gegebenem" hinaus kaum Schwierigkeiten; es geht um rim Charakter interpretative, d.h. perspektivische, konstruktbildende, ein- und auslegende Vorgänge <-); (c) den kategorialisierenden Status der Interpretation $_{1}$-Ebene (wobei die formierten Welten nicht als aus dem Nichts geschöpfte Welten angesehen werden können); (d) daß bei aller Betonung der Pluralität der Welten die Rede von >der einen Welt $<$, recht verstanden, sehr wohl guten Sinn macht, und daß die Pluralitätsthese unterschiedlich ausfällt, je nachdem, um welche Ebene der Interpretationsver-

9 Zu ersten Überlegungen in diese Richtung vgl. Verf., Wahrheit als Interpretation, in: Krisis der Metaphysik. Festschrift für Wolfgang Müller-Lauter, hg. von G. Abel/ J. Salaquarda, Berlin/New York 1989, 331-363; und: Zum Wahrheitsverständnis jenseits von Naturalismus und Essentialismus, in: Perspektiven des Perspektivismus. Gedenkschrift zum Tode von Friedrich Kaulbach, hg. von V. Gerhardt/N. Herold, Würzburg 1993, 313-335.

10 Jenseits von Gegebensein und Machen. Interpretationspluralistischer Monismus als Alternative zu Abels Weltenvielfalt, im vorliegenden Heft der Deutschen Zeitschrift für Philosophie, 867 ff. 
hältnisse es sich handelt; (e) daß, da es unterschiedliche Interpretation ${ }_{1}$-Systeme geben kann, von einer Pluralität so vieler Welten auszugehen ist, wie es kohärente (die Bedingungen der empirischen Gültigkeit einbeziehende) Interpretationen ${ }_{1}$ gibt (was nicht heißt, daß es so viele Welten wie mögliche Interpretationen gibt; denn nicht alle Interpretationen sind welt-formierende Interpretationen); (f) daß die Rede von der Vielheit wirklicher Interpretationswelten und die Rede von seiner، Welt zusammengehören.

\section{Interne und konditionale Pluralität}

Die Interpretationsphilosophie verteidigt, wie betont, das Konzept einer internen und konditionalen Pluralität der Interpretationen und auch der Welten. ${ }^{11}$ Dabei lassen sich „Gründe“ der Pluralität von „Arten“ der Pluralität unterscheiden. Die Gründe können ausgezeichnet werden u.a. entlang des interpretativen Charakters der Symbolsysteme, im Zuge der Unbestimmtheit der Übersetzung und der Interpretation oder im Zusammenhang der Individuation von Objekten und Ereignissen. Als Arten der Pluralität ergeben sich daraus u.a. eine Pluralität der Welten, der Wahrheiten und der Referenten bzw. der Ontologien. Interne Pluralität läßt sich, so die These, unabhängig von reduktionistischen Annahmen formulieren und erweist sich als unvermeidlich und als nicht-eliminierbar. „Intern“ heißt die Pluralität, da es u.a. der Interpretationscharakter der kategorialisierenden Interpretation $_{1}$-Systeme selbst ist, der >von innen her < die Möglichkeit des Auch-anders-seinkönnens, der Alterität und Variabilität mit sich führt. „Konditional“ heißt die Pluralität im Sinne der These, daß es viele Interpretations-Welten geben muß, wenn es denn überhaupt eine geben können soll. Die eine Interpretations-Welt, in der wir leben, ist in diesem Sinne stets bereits auf andere Interpretations-Welten bezogen, um die eine sein zu können, die sie für uns ist. (Das heißt freilich nicht, daß wir in vielen disjunkten Welten gleichzeitig leben und uns auf sie verstehen müßten.)

Vor diesem Hintergrund ist auch der von Gunnarsson (872) richtig gesehene Punkt zu verstehen, daß die Vielheit der Interpretationswelten mit einem bestimmten Sinn der Rede von >einer Welt $<$ zu verbinden ist (und daß diese Unterstellung in der Interpretationsphilosophie eine wichtigere Rolle spielt als im Konstruktionalismus Goodmans). Die entscheidende Frage jedoch lautet, wie diese Annahme zu verstehen und wo genau sie anzusiedeln ist. Ich fasse sie als eine interne Präsupposition auf der Ebene eines Welt-, Fremd- und Selbstverständnisses und als interne sinnlogische Präsupposition unseres Sprechens, Denkens und Handelns auf; - aber eben als eine Präsupposition, nicht, wie Gunnarsson im IPM intendiert, als $>$ Die Ein- und Dieselbe Welt $<$, die als eine von allen bestimmten Interpretationen $_{1}$,unabhängige Welt“ (875) konzipiert werden soll. Gunnarssons Vorstellung ist, daß diese >Ein und Dieselbe Welt „in unterschiedlichen Interpretationen zum Ausdruck (kommt)“ (873, Anm. 17). Da der IPM nicht eine Welt meinen soll, die als ein ,nichtinterpretiertes Etwas“ „hinter aller Interpretation“ (873 f.) steht, ist selbst eine Welt, wie die im IPM gedachte, von der angesprochenen konditionalen Pluralität bereits abhängig und muß

11 Zu den Einzelheiten vgl., neben Verf.: Interpretationswelten. Gegenwartsphilosophie jenseits von Essentialismus und Relativismus, Frankfurt/M. 1993 (Register: Pluralismus; Pluralität), Verf., Interne Pluralität, a.a.O.; und: Interpretationsphilosophie, in: Allgemeine Zeitschrift für Philosophie, 13 (1.988), insbes. $83 \mathrm{f}$. 
diese in Anspruch nehmen. Soll der IPM akzeptabel sein, so müßte gezeigt werden, daß die Annahme >Der Ein und Derselben Welt c konditional ist, wir es mithin gar nicht mit einer bestimmten Welt und nicht mit bestimmten Interpretationen zu tun haben könnten, wenn nicht > Die Eine Welt< angenommen wäre. Ein solcher Nachweis liegt, soweit ich sehe, nicht vor. Die Annahme des IPM erscheint als synkategorematisch zur konditionalen Pluralität, nicht umgekehrt.

\section{Schwierigkeiten des IPM}

Gunnarsson räumt ein (873), daß „,keine einzige richtige Interpretation (oder Kombination von Interpretationen) (existiert), die ausdrücken könnte, was für eine Welt die sein und dieselbe Welt< ist“, und er schlägt vor (873), genau diesen Umstand als eine ,zentrale Erkenntnis" über den Begriff dieser Welt anzusehen. Mit dieser Konzeption sind eine Reihe von Schwierigkeiten verbunden, von denen ich stenogrammartig die wichtigsten zu bedenken gebe:

(i) Daß es keine richtige und vollständige Interpretation der Welt gibt, heißt, daß es keine definitive und allgemein verbindliche Weltinterpretation gibt. Diesen Umstand sollten wir nicht zu einer verborgenen Eigenschaft >Der Einen Welt< hypostasieren, sondern dort belassen, wo er auftritt: auf der Ebene der Unabschließbarkeit der Interpretationen. Und wenn es zwei oder mehrere kategorialisierende Interpretation ${ }_{1}$-Systeme gibt, die weder aufeinander reduzierbar noch auf ein beiden gemeinsames Drittes zurückführbar sind, dann sprechen wir, mit Goodman, besser von zwei oder mehreren Welten. Angesichts des kategorialisierenden Status mehrerer untereinander unvereinbarer Interpretation ${ }_{1}$ Systeme $^{-}$ macht es unter kritischem Vorzeichen kaum Sinn, von >Ein und Derselben Welt $<$ zu sprechen, von der die Interpretation ${ }_{1}$-Kategorialisierungen inkompatible Kategorialisierungen sind. (ii) Gezeigt werden müßte, daß die Annahme >Ein und Derselben Welt $<$ intern unvermeidlich, nicht-eliminierbar und konditional für das Auftreten unterschiedlicher, sogar inkompatibler, jedoch gleichermaßen richtiger Interpretationen ist, daß wir ohne Annahme Der Einen Welt überhaupt nicht zu Individuationen, Identifikationen, Re-Identifikationen und raum-zeitlichen Lokalisierungen von Gegenständen kommen könnten. (iii) Wenn der IPM zuträfe, dann wäre Skeptizismus selbst noch in den bestgesicherten Fällen unserer Interpretationen ${ }_{3+2}$ jederzeit möglich. Es könnte sich in Wirklichkeit stets noch von Grund auf anders verhalten, als selbst in den bestmöglichen unserer Interpretationen artikuliert. Zudem könnte dieser Aspekt dazu führen, uns unsere vertraute Welt fremd werden zu lassen. (iv) Ein Vorzug der interpretationsphilosophischen Konzeption liegt darin, daß sie beides zusammenbringen kann, die konditionale Pluralität und die Einheit eines jeden Weltverständnisses im Sinne der internen Präsupposition unseres Sprechens, Denkens und vor allem unseres Handelns. (v) Dem IPM zufolge ist , Die Eine Welt $<$ von der Art, daß sie viele Interpretationen zuläßt, in denen sie ,zum Ausdruck komme“. Nicht von ungefähr bleibt diese Ausdrucksbeziehung völlig ungeklärt. Was könnte man auch über sie sagen, da $>$ Die Eine Welt< doch von allen bestimmten Interpretationen ${ }_{1}$ unabhängig sein soll. (vi) Die Funktion der Rede von ein und derselben Welt wird darin gesehen, ,unsere interpretatorischen Bestrebungen zu vereinheitlichen“ (874). Doch die Annahme, daß unsere verschiedenen Interpretationen alle von >Ein und Derselben Welt< handeln, gerät in dem Moment in Schwierigkeiten, wo es sich um untereinander unvereinbare, inkompatible, jedoch glei- 
chermaßen richtige Interpretationen handelt, die alle die Kohärenzanforderungen unter Einschluß der empirischen Gültigkeit gleichermaßen gut erfüllen. Können dies dann auch Interpretationen ,Ein und Derselben Welt< sein? Wie sollte das möglich sein, wenn, wie Gunnarsson zugesteht, eine logische Kluft zwischen Interpretation ${ }_{1}$ und Welt, zwischen Interpretativität und Faktizität, nicht mehr expliziert werden kann. Man kann nicht beides zugleich haben: die >Ein und Dieselbe Welt « im Sinne des IPM und untereinander unvereinbare alternative Interpretation ${ }_{1}$-Systeme.

\section{Einheit und Andersheit}

Wird „Welt“ im weitesten Sinne als der Inbegriff der Erscheinungen bzw. dessen verstanden, wovon die Rede sein kann, dann ist, mit einer Formulierung Simons (863) gesprochen, zu beachten, daß , alles, > wovon < die Rede ist, in einer Welt ist, die meine Welt ist“. Die Grenzen dieser > Einen Welt $<$, meiner und mit anderen (z.B. in öffentlichen Kommunikations- und Wahrnehmungssituationen sowie in Handlungskontexten) gemeinsam geteilten und in diesem Sinne auch gemeinschaftlichen Welt können sich verschieben, andere werden, erweitern oder verengen. Kommen darin andere Prinzipien der Individuation, der Identifikation und der raum-zeitlichen Lokalisierung ins Spiel, bedeuten solche Veränderungen den Übergang in eine veränderte, in eine andere Welt. Man bewegt sich von da an in einer anderen, jedoch ihrerseits wiederum >einen<, wieder um >meiner $<$ Welt. Doch diese >eine und meine Welt $<$ ist nicht die im IPM konzipierte >Die Eine Welt $<$. Denn sie ist gerade nicht unabhängig von allen bestimmten Interpretationen ${ }_{1}$. Da >meine $<$ bzw. >unsere Welt immer schon eine interpretation ${ }_{1}$-formierte und interpretation $1^{-}$, begrenzte $^{\prime}$ Welt ist, ist diese reine und meine bzw. unsere Welt < intern mit der Möglichkeit anderer Welten im Sinne des internen und konditionalen Auch-anders-sein-könnens meiner bzw. unserer Welt verbunden. Man sieht, daß und in welchem Sinne Einheit und Andersheit der Welt in dieser Konzeption zusammengehen. Dies zeigt auch, daß es im Verhältnis von Interpretativität und Faktizität auf der Interpretation ${ }_{1}$-Ebene nicht mehr um Prioritätsverhältnisse im älteren Sinne gehen kann.

\section{HI. Zu Thomas Bonk}

Der Beitrag von Thomas Bonk ${ }^{12}$ stellt die Frage, ob im Rekurs auf die Interpretationen die lang ausstehende Widerlegung des Skeptizismus gelingt. Der Beitrag wirft wichtige Fragen hinsichtlich des Verhältnisses von Interpretation und Erfahrung und vor allem von Interpretations-Praxis und erkenntnistheoretischer Gewißheit auf.

\section{Philosophischer Skeptizismus}

$\mathrm{Zu}$ beachten ist zunächst, daß ich in den „Interpretationswelten“ keine erkenntnistheoretische „Widerlegung“ oder „Eliminierung“ der Skepsis betreibe, sondern vielmehr daran interessiert bin, dem internen Skeptiker eine pragmatisch „,zufriedenstellende“ Antwort zu

12 Erfahrung und Skepsis. Anmerkungen zum „Interpretationismus", im vorliegenden Heft der Deutschen Zeitschrift für Philosophie, 879 ff. 
geben. Der Skeptiker wird so stark gemacht wie nur möglich. Seine Zweifel führen in die Probleme der Erkenntnisfragen und zugleich in den interpretatorischen Charakter des menschlichen Welt-, Fremd- und Selbstverständnisses. Da der Interpretationscharakter nicht nur eingeräumt, sondern leitend wird, ist eine den Skeptiker zufriedenstellende Situation skizzierbar. Im Kern handelt es sich dabei um einen Rekurs auf pragmatisch und praktisch erforderte interpretative Präsuppositionen, nicht mehr um Seinsannahmen, Wahrheitsgarantien oder Letztbegründungen. Die gesamte Seins- und erkenntnistheoretische Gewißheits-Architektur wird vielmehr zurückgelassen. Voraussetzung dafür ist, daß das „Wissen“ im Sinne Kants als einer der drei Modi des „Fürwahrhaltens“ (Meinen, Glauben, Wissen) gefaßt und dieses Netzwerk des Fürwahrhaltens insgesamt in seinem internen Zusammenhang mit dem fraglosen Funktionieren der eingespielten Interpretations-Praxis gesehen wird, aus der heraus und auf die hin die semantischen, die pragmatischen und die praktischen Merkmale unserer Zeichen und Handlungen bestimmt werden.

\section{Interpretations-Praxis und Gewißheit}

Bonk sieht (883 ff.) die zentrale Stellung der Interpretations-Praxis ${ }^{13}$ deutlich, auch in ihrem Zusammenhang mit der Skepsisproblematik. Gegenüber seiner Konzentration auf die Frage der Bereitstellung einer anti-skeptischen Gewißheit sind jedoch zwei Begriffe von „Gewißheit" zu unterscheiden: die sepistemologische bzw. erkenntnistheoretische Gewißheit< und die sinterpretationspraxis-bezogene, praktische Gewißheit . Die erste Art der Gewißheit ist nicht zu haben und bleibt jederzeit skeptisch angreifbar. Ein solches Konzept der Gewißheit habe ich, anders als Bonk zu vermuten scheint, nicht vertreten. Die zweite Art der Gewißheit meint die mit dem fraglos funktionierenden Eingespieltsein einer Interpretations-Praxis (Lebenspraxis) intern gegebenen Selbstverständlichkeiten. Diese haben ihrerseits keine weiteren Grundlagen als eben unsere Interpretations- und Handlungsweisen selbst (was freilich nicht im Sinne einer Wahrheitsgarantie oder einer Letztbegründung mißverstanden werden darf). Analog zu Wittgensteins Formulierung "So handle ich eben“14 könnte diese interpretations-praktische Gewißheit in der Formulierung „So interpretiere ich eben" zum Ausdruck gebracht werden. Darin zeigt sich zugleich der elusive und der ineffable Charakter dieser praktischen Sicherheit, dieses praktischen Vertrauens in das, was man sagt, denkt und tut, wenn man es so sagt, denkt und tut, wie man es sagt, denkt und tut. Ohne diese interpretations-praktische Gewißheit würden wir gar nicht ins wirkliche Sprechen, Denken und Handeln eintreten. Diese Art der Gewißheit spielt bei Wittgenstein ${ }^{15}$ eine grundlegende Rolle, da sie die theoretische Wahrheitssuche durch die Frage der praktischen Gewißheit im Sprechen, Denken und Handeln ablöst. Die Selbstverständlichkeiten im Fürwahrhalten sind auch bereits vorausgesetzt, wenn überhaupt ein Zweifel geäußert werden kann. Mit Wittgenstein gesprochen gilt: „Der Zweifel kommt nach dem Glauben“16 (als dem zweiten der drei Kantischen Modi des Fürwahrhaltens) und: „Der vernünftige

13 Vgl. Verf., Interpretation und Realität, a.a.O., 286-288.

14 L. Wittgenstein, Philosophische Untersuchungen, Nr. 217.

15 Vgl. vor allem L. Wittgenstein, Über Gewißheit, hg. von G. E. M. Anscombe und G. H. von Wright, Frankfurt/M. 1970.

16 L. Wittgenstein, Über Gewißheit, Nr. 160. 
Mensch hat gewisse Zweifel nicht ${ }^{\prime \prime} \cdot{ }^{17}$ Entsprechend ist die sinterpretations-praktische Gewißheit $<$ nicht etwas, das wir erreichen müssen, auf das wir aus sind. Sie ist vielmehr fraglos da (oder nicht). Das Projekt einer >Suche nach der Gewißheit< ist gerade das, was zurückzulassen ist. Das interpretations-praktische Vertrauen läßt sich demgemäß auch nicht, herstellen' (gar dadurch, daß der Skeptizismus durch die Bereitstellung von Wahrheit garantierenden Sätzen widerlegt werden soll). Es kann nicht mehr um das ältere metaphysische Projekt der Erkenntnistheorie im Sinne der Sicherung metaphysischer Wissensgehalte gehen. Das heißt natürlich nicht, daß Erkenntnisfragen damit verschwinden. Aber der Sinn dessen, was wir da meinen, hat eine Veränderung erfahren. Spannend ist jetzt u.a. die Frage nach dem Verhältnis von interpretations-praktischer Gewißheit $_{1}$ und Gewißheit ${ }_{3}$, mithin, so könnte man sagen, das Verhältnis von Vertrauen und Wahrheit.

In Sachen Gewißheit wie in puncto zufriedenstellende Antwort auf die Herausforderungen des philosophischen Skeptizismus bewegt sich der Interpretationismus im Rahmen der interpretationspraktischen Gewißheit ${ }_{1}$. Bonk scheint mir den wichtigen Unterschied nicht angemessen zu beachten, der, wie wir in Aufnahme der Stufungen des Interpretationsmodells sagen können, zwischen der "Gewißheit “ ( = der erkenntnistheoretischen Gewißheit auf der Interpretation ${ }_{3}$-Ebene) und der „Gewißheit ${ }^{\text {“ ( }}$ ( = dem der Interpretation -Praxis $_{1}$ eigentümlichen praktischen Vertrauen in das, was man sagt, denkt und tut) besteht.

\section{Welthaltigkeit der Zeichen- und Interpretationswelten}

Im Prozeß der Gegenstandsformation, der von den Empfindungsmaterialien bis hin zu expliziten Objekten und Ereignissen reicht, sehe ich „Interpretations-Ketten " am Werke. ${ }^{18}$ Bonk betont mit Recht $(879 ; 881)$ deren Wichtigkeit und ihren nicht-reduktionistischen Charakter (880). Von der „Welthaltigkeit“ der Zeichen- und Interpretationswelten kann ihm zufolge (881) nur an der Stelle des Inputs der Empfindungsmaterialien die Rede sein. Das ist ein enger Begriff von Erfahrung und Welthaltigkeit. Die Interpretationsphilosophie ist hier eher in der Nähe der Kantischen Auffassung zu sehen, derzufolge es sich bei >Objekten um eine Vereinigung des Mannigfaltigen einer Anschauung in einem Objekt, z.B. dem Objekt > Tisch <, und bei > Erfahrung < um den Inbegriff aller durch die Sinne gegebenen und durch den Verstand gedachten Erkenntnisse anschaulichen Gehalts handelt. Darüber hinaus gilt es, zwei weitere Aspekte zu beachten.

(a) Welthaltigkeit kommt gerade auch über die fraglos eingespielte Interpretations-Praxis ins Spiel. Wenn Zeichen nur dann semantische Merkmale (Bedeutung, Referenz, Wahrheits- und Erfüllungsbedingungen) haben, wenn ihnen eine Interpretations-Praxis bereits vorausliegt, in der diese Merkmale umgrenzt werden, dann sind diejenigen Zeichen, nach deren Bedeutung und Referenz nicht eigens gefragt wird, intern zugleich die wirklichkeitsund realitätshaltigsten Zeichen überhaupt. ${ }^{19}$ Man fragt dann nicht noch einmal, ob den Zeichen auch tatsächlich etwas Wirkliches/Reales entspricht. Im Gebrauch der Zeichen

17 Ebd., Nr. 220.

$18 \mathrm{Zu}$ einer interpretationistischen Konzeption der Erfahrungserkenntnis vgl. Verf., Theorie, Beobachtung und Wirklichkeit, in: Theorien, Modelle, Tatsachen. Konzepte der Philosophie und der Wissenschaften, hg. von H. J. Sandkühler, Frankfurt/M. 1994, 9-29.

19 Vgl. Verf., Interpretation und Realität, a.a.O., 284 ff. 
sind wir aus praktischen Gründen an einem bestimmten Punkt sinnlogisch gezwungen, von der innersprachlichen Intension zur expliziten Referenz auf äußere Objekte überzugehen, d.h. zu unterstellen, daß es so etwas, wovon da die Rede ist, auch tatsächlich gibt. (b) Welthaltigkeit der Interpretations-Praxis zeigt sich auch daran, daß, wie oben (in dem Kommentar zu Simon, Punkt 4) bereits betont, die Fähigkeit, eine semantische Charakterisierung einer Sprache zu geben, an die Fähigkeit geknüpft ist, etwas über die zugrundegelegte Welt sagen zu können.

\section{Zu Geert-Lueke Lueken}

Der Beitrag von Geert-Lueke Lueken ${ }^{20}$ möchte das interpretationsphilosophische Projekt durch den Nachweis kritisieren, daß (a) sinnkritische Argumentation verfehlt werde, (b) der erweiterte Gebrauch des Wortes „Interpretation“ dessen unterscheidende Kraft gefährde und (c) das Skepsis-Problem ein praktisch irrelevantes Problem sei. Eine genaue Prüfung zeigt jedoch, daß keine dieser Behauptungen zutrifft.

\section{Sinnkritische Argumentation}

Philosophische Probleme werden in der Regel nicht beweis-deduktiv, gelöst', sondern durch die Angabe derjenigen sinnkritischen Bedingungen auf-gelöst, zum Verschwinden gebracht, unter denen sie nicht mehr entstehen. So verfährt die Interpretationsphilosophie (z.B. dann, wenn sie das vertikale Stufenmodell der Interpretation einführt, um mit Problemen umgehen zu können, die in der horizontalen Ebene nicht aufgelöst werden können). Es mutet daher ein wenig eigentümlich an, wenn Lueken mich unter den Verdacht fehlgehender sinnkritischer Argumentation stellen möchte. Seine Beispiele lösen diesen Verdacht nicht ein. ${ }^{21}$ Ich verweise daher einfach auf eine genauere Lektüre der entsprechenden Passagen der "Interpretationswelten“.

\section{Rekonstruktionen}

Im rekonstruierenden Teil seines Beitrags (892-898) läßt Lueken sich auf das Grundwort „Interpretation“ und auf das Stufenmodell der Interpretation ein. Anzumerken ist freilich,

20 „Alles, was so ist, könnte auch anders sein“. Zu Günter Abels „Interpretationswelten“, im vorliegenden Heft der Deutschen Zeitschrift für Philosophie, 889 ff.

21 Beispiel 1: Die Charakterisierung des Verifikationsprinzips des Logischen Empirismus wird von Lueken (890) nicht korrekt wiedergegeben und der Kern des Problems, das in den Kapiteln 6-9 behandelt wird: das Verhältnis zwischen seliminativem< und >analytischem< Verifikationismus, wird nicht einmal erwähnt. Beispiel 2: In bezug auf das Verhältnis von eliminativem Verifikationsprinzip und Skeptizismus wird nicht gesehen, daß der interne Skeptizismus $>$ konditional $<$ für das Verifikationsprinzip ist - nicht umgekehrt. Beispiel 3: Im Rahmen der Gehirne-im-Tank-Fiktion wird mir irrigerweise unterstellt, ich wolle so etwas wie einen ,ppositiven“ Nachweis dafür erbringen, daß wir keine Gehirne-im-Tank sind. Beispiel 4: Die Pointe der Goodmanschen Argumentation in Sachen Pluralität der Welten ist nicht, daß wir von fertigen verschiedenen Welten ausgehen, sondern daß wir kategorialisierenden Versionen, die richtig, jedoch untereinander unvereinbar sind, sinnvollerweise verschiedene Welten zuordnen. 
daß das Grundanliegen des interpretationsphilosophischen Ansatzes bei Lueken nicht wirklich im Vordergrund steht: daß es nämlich darum geht, den interpretativen (und das heißt: den perspektivischen, konstruktbildenden, projizierenden, konjekturalen, wertschätzenden, präferenzierenden, ein- und auslegenden) Charakter des menschlichen Welt-, Fremd- und Selbstverständnisses/verhältnisses herauszuarbeiten und zum Leitfaden der Betrachtung zu machen. Es geht darum, daß die entsprechenden Grundvorgänge als >im Charakter interpretative Vorgänge < konzipiert werden. Das ist der wesentliche Zweck, der verfolgt wird. Und um die unterscheidende Kraft des Konzepts der Interpretation in dieser Erweiterung nicht nur aufrechtzuerhalten, sondern zu steigern, wird, wie Lueken (893) richtig sieht, der Ausdruck im Stufenmodell der Interpretation intern differenziert. Je feinkörniger die internen Differenzierungen sind, desto deutlicher treten die Unterschiede zwischen den verschiedenen Ebenen, Hinsichten und Vorgängen hervor. Die detaillierten Unterscheidungen können (wie ich bereits in der Einleitung der „Interpretationswelten“, 15, Anm. 1, mit Hinweis auf weitere Texte, betont habe) je nachdem, was als das Wesentliche gilt und welches der damit verfolgte Zweck ist, erforderlich oder auch entbehrlich sein.

Zur Rekonstruktion der einzelnen Typen der Interpretation bei Lueken möchte ich stichwortartig einige Aspekte hervorheben oder ergänzen: (i) Die Einsicht, daß es den unterschiedlichen Prozessen des Verstehens, Erkennens und Handelns gemeinsam ist, ihnen eine ,als-Struktur“ (894) unterlegen zu müssen, kann dahingehend akzentuiert werden, daß das >Ansehen-als der grundlegende Vorgang menschlichen Welthabens ist. (ii) Eine in diesem Zusammenhang wichtige Frage ist, woher wir denn wissen können, daß es alternative Interpretationen geben könnte, solange es keine expliziten Alternativen gibt (894). Die Antwort lautet: Die Rede von Alternativen, Alterität und Pluralität darf, wie bereits in den Repliken auf Gunnarsson und Simon betont, nicht >extern<, sondern muß >intern $<$ und darüber hinaus skonditional verstanden werden. Lueken verkennt diesen Punkt, wenn er mir später (900) sogar unterstellt, ich müsse theoretisch eine Art externen Gottesgesichtspunkt einnehmen. (iii) Wichtig finde ich den von Lueken (897) mit Bezug auf Wittgenstein betonten Hinweis auf die unterschiedlichen Funktionen von Zeigewörtern und Zeigehandlungen je nachdem, ob es um Lehr- bzw. Lernsituationen oder um hinweisende Erklärungen bzw. Definitionen geht. Letzteren traue ich, wie Lueken richtig vermutet, mit Wittgenstein, nicht sehr viel zu; ihren Einsatz im „hinweisenden Lehren“ dagegen veranschlage auch ich sehr hoch.

\section{Skepsis-Möglichkeit}

Unzutreffend ist, was Lueken (898-901) über den Sinn meiner Rede von „Skepsis-Möglichkeit" ausführt. Zwar sieht er (899) richtig, daß der >interne Skeptizismus « sich nicht darauf stützt, daß reale Unterschiede zwischen realen „Orientierungssystemen“ bestehen (welchen Aspekt Lueken offenkundig favorisiert). Denn in der Tat hat es philosophisch primär um die Frage zu gehen, wie Skepsis logisch möglich ist. Lueken sieht nun den internen Skeptizismus allein auf der Gedankenfigur beruhen, „daß alles ganz anders sein könnte“ (899), und er stützt sich dabei auf meinen Satz „Alles, was so ist, könnte auch anders sein“ („Interpretationswelten“, 504; 92). Dazu sei folgendes angemerkt:

(i) In bezug auf den Satz des Auch-anders-sein-könnens mißversteht Lueken (898) das ,alles“ als ein ,alles schlechthin und auf einmal“. Die Rede von ,alles“ ist jedoch zu para- 
phrasieren als: „ein jedes Ding“ im Sinne von „ein jedes individuiertes und raum-zeitlich lokalisiertes Objekt und Ereignis".22 Das „alles“ ist nicht das Totalitäts-"alles“. Diese Lesart ist systematisch auszuschließen. Denn der Ausgangspunkt des interpretationsphilosophischen Ansatzes liegt in Endlichkeit, finiten Zeichen und finitisierenden Zeichenfunktionen.

(ii) Wollte man statt der These des Auch-anders-sein-könnens den Satz verteidigen „Alles, was so ist, kann nicht anders sein", so wäre man damit auf uneinlösbare Beweislasten verpflichtet. Wir müßten voraussetzen, daß es sowohl eine essentialistische und apriorische Ordnung der Dinge als auch ein intrinsisches und definitives vorab-Festgelegtsein der semantischen Merkmale der Zeichen (in ihren verständigungs-orientierten wie in ihren gegenstands-konstitutiven Funktionen) gibt. Es ist jedoch keine Philosophie in Sicht, die diese Voraussetzungen unter kritischem Vorzeichen verteidigen könnte. Erinnert sei an Wittgenstein, der betont, „daß kein Teil unserer Erfahrung auch a priori ist. Alles, was wir sehen, könnte auch anders sein" $" 23$.

(iii) Die Frage (899), von wo aus die Möglichkeit des Auch-anders-sein-könnens und damit die Skepsis-Möglichkeit überhaupt in den Blick komme, beantworte ich also gerade nicht, wie Lueken (900) irrigerweise meint, aus einer „,absoluten Beobachterperspektive“. Die Antwort lautet einfach: Die Skepsis-Möglichkeit ergibt sich im Zuge der internen Alterität und Pluralität ${ }^{24}$ der aus der Interpretations-Praxis heraus und auf diese hin erfolgenden welt-formierenden Kategorialisierungen selbst, im Zuge mithin dessen, was es heißt, unter Endlichkeitsbedingungen über ein Welt-, Fremd- und Selbstverständnis zu verfügen.

(iv) Von hier aus wird zugleich deutlich, daß und in welchem Sinne die Skepsis-Möglichkeit interne Alternativen eröffnet und dem Skeptiker doch zugleich auch im Rekurs auf die Interpretations-Praxis (die bei Lueken nicht zur Sprache kommt) eine zufriedenstellende Antwort in dem Sinne gegeben werden kann, wie dies in der Replik auf Thomas Bonks Beitrag skizziert wurde.

(v) Der Unterschied zu Luekens Auffassung läßt sich auch an der Frage der Unbestimmtheit der Referenz, der Übersetzung und der Interpretation verdeutlichen, auf die Lueken selbst sich stützen zu können glaubt (900 f.). Auf den gegenwärtigen Zusammenhang bezogen ist der Clou der Quineschen Unbestimmtheitsthese ein dreifacher ${ }^{25}$ : Erstens (a) kann die Unbestimmtheitsthese deshalb ohne reduktionistische Annahmen formuliert und als nicht-eliminierbar erwiesen werden, weil wir als endliche Geister in Verständigungsverhältnissen von einer extramundanen Perspektive systematisch abgeschnitten sind. Zweitens (b) benötigt man, gerade weil man keinen externen Standpunkt einnehmen kann, im Übersetzen und Verstehen interpretatorische Übersetzungs- und Interpretationshypothesen (die Quine „analytische Hypothesen“ nennt), die über alle möglichen empirischen Daten und über alle möglichen Sprachdispositionen hinausgehen. Die interne Unbestimmtheitsthese besagt dann: Es kann mehrere alternative Systeme solcher Interpretati-

22 Vgl. Verf., Interpretation und Realität, a.a.O., 277-282.

23 L. Wittgenstein, Tractatus, Nr. 5.634.

$24 \mathrm{Vgl}$. Verf., Interne Pluralität, a.a.O.

25 Vgl. im einzelnen Verf., Unbestimmtheit der Interpretation, in: Distanz im Verstehen. Zeichen und Interpretation II, hg. von J. Simon, Frankfurt/M. 1995, 43-71. 
onshypothesen geben, die untereinander inkompatibel, jedoch empirisch äquivalent und mit allen Sprecherdispositionen gleichermaßen gut vereinbar sind. Dies gilt nicht nur fremden Sprachen gegenüber, sondern auch innerhalb einer Sprache. Auch in einer gegebenen Sprache können die Denotationen der Terme permutieren, und dies bliebe doch immer noch mit allen möglichen Sprachdispositionen verträglich. Drittens (c) besteht der entscheidende Punkt nicht darin, daß wir nicht sicher sein können, ob die gewählte Interpretationshypothese richtig ist, ,but that there is not even (...) an objective matter to be right or wrong about" 26 . Die Unbestimmtheit ist keine Unbestimmtheit von Fakten und Tatsachen, d.h. Unbestimmtheit würde auch dann noch bestehen, wenn wir die bestmögliche Theorie über die gesamte Natur besäßen. Diese Punkte sind auch für meine Auffassungen entscheidend. Sie werden von Lueken jedoch verkannt, wenn er schreibt (900): „Bestimmtheit durch eine Interpretationswelt wird nur dann zu einem Argument für die Möglichkeit alternativer Bestimmbarkeit, wenn man von außen so etwas wie objektive Unbestimmtheit behauptet. Genau dies scheint Abel tun zu wollen. "Offenkundig geht dies an meiner Argumentation vorbei. Die philosophisch spannende Frage lautet vielmehr: Wie ist der Zusammenhang zu denken zwischen der nicht-eliminierbaren Unbestimmtheit sowie der mit dieser intern verbundenen Möglichkeit alternativer Interpretationen und der interindividuell gelingenden sowie welt-formierenden Verständigung?

Abschließend möchte ich auf einen Sinn des Satzes „Alles, was so ist, könnte auch anders sein" hinweisen, der für den interpretationsphilosophischen Ansatz kennzeichnend ist. Gemeint ist die bereits betonte zentrale Rolle des Ansehens-als. Wenn wir nicht mehr von einer essentialistischen oder apriorischen Ordnung der Dinge ausgehen können, und wenn symbolisierende Zeichen ihre semantischen Merkmale nicht von einem ,hinter' den Zeichen stehenden metaphysischen oder apriorischen Signifikat vorab und allgemein verbindlich zugewiesen bekommen, dann kommt offenkundig alles darauf an, wie die Dinge angesehen werden. Das ist, wie betont, nicht beliebig. Aber es schließt doch ein, daß alles, was so-und-so angesehen wird, im Prinzip auch anders angesehen werden könnte. Diese Formulierung, in der "sein" durch ,ansehen“ ersetzt wird, ist der Sache nach dem interpretationsphilosophischen Ansatz noch angemessener als die oben erörterte Formulierung des Auch-anders-sein-könnens. Darin wird auch in der Darstellung dem Sachverhalt Rechnung getragen, daß es in der Interpretationsphilosophie nicht um Seins-, sondern um Interpretationsverhältnisse geht. Dies gilt natürlich auch für philosophische Debatten. Man kann die Dinge, um die es geht, auch anders sehen. Die Interpretationsphilosophie beläßt anderen ihre anderen Interpretationen.

Prof. Dr. Günter Abel, Technische Universität Berlin, Institut für Philosophie, Ernst-ReuterPlatz 7, 10587 Berlin

26 W. V. O. Quine, Word and Object, Cambridge, Mass. ${ }^{13} 1983,73$. 